Retrospective Evaluation

\title{
Combining Ultrasonography and Electromyography for Botulinum Chemodenervation Treatment of Thoracic Outlet Syndrome: Comparison with Fluoroscopy and Electromyography Guidance
}

Sheldon E. Jordan, MD1', Sam S. Ahn, MD², and Hugh A. Gelabert, MD³

From: ${ }^{1} U C L A$ Department of Neurology, Los Angeles, CA; ${ }^{2}$ University Vascular Associates, Los Angeles, CA; and Saint John's Health Center/ John Wayne Institute, Santa Monica, CA

Dr. Jordan ${ }^{1}$ is with the UCLA Department of Neurology. Dr. Ahn² is with University Vascular Associates and is attending surgeon at the UCLA Department of Surgery, Gonda Vascular Center, Los Angeles, CA

Dr. Gelabert ${ }^{3}$ is with the UCLA Department of Vascular Surgery, Los Angeles, CA

Address correspondence: Sheldon E Jordan, MD Neurological Associates of West Los Angeles

2811 Wilshire Blvd, Suite 790

Santa Monica, California 90403

E-mail: shellyj@aol.com

Disclaimer: No external funding for this article was provided. Conflict of interest: None Manuscript received: 04/30/2007 Accepted for Publication: 05/28/2007

Free full manuscript: www.painphysicianjournal.com
Background: Botulinum chemodenervation has been increasingly used for treating conditions characterized by muscular pain and dystonia. Complication rates commonly exceed 10 percent in published accounts due to an inadvertent spread of toxin. Various techniques of precision targeting have been described to minimize undesirable toxin effects. The present study reports on a clinical experience combining ultrasonography and electromyography in order to demonstrate how favorably this approach compares to previously described techniques in terms of minimizing complications while maintaining efficacy.

Design: Retrospective case series

Methods: The present study is a retrospective clinical analysis of patients treated with botulinum toxin using 2 different combined targeting techniques; one using ultrasonography and electromyography and the other using fluoroscopy and electromyography.

Results: Combined ultrasonography and electromyography was used in 77 of 245 procedures; in 168 procedures, fluoroscopy and electromyography was used. There were no complications with ultrasonography guided procedures; the complication rate for combined fluoroscopy and electromyography was 1.8 percent (3/168; Fisher exact $p=$ 0.3206). For combined ultrasonography and electromyography, after 70 out of 77 procedures $(91 \%)$ there was a good outcome compared to 136 out of $168(81 \%)$ after procedures utilizing a combination of fluoroscopy and electromyography (Fisher exact $p=$ $0.331)$.

Conclusions: There was no significant difference in complication rate or successful outcomes comparing the 2 forms of imaging guidance when targeting muscles for botulinum injection. Since outcomes are comparable, other factors such as cost and radiation exposure may be considered in choosing which imaging modality to use.

Key words: thoracic outlet syndrome, brachial plexus, botulinum toxin, ultrasound

Pain Physician 2007; 10:541-546 
The diagnosis and treatment of Thoracic Outlet Syndrome remains a controversial subject. Nevertheless, patients present in pain medicine practices with cervical brachial pain and no convincing evidence of cervical radiculopathy, ulnar neuropathy, or Carpal Tunnel Syndrome. For those patients with cervical brachial pain who fail to improve with physical therapy and ergonomic adjustments, offering a minimally invasive treatment option would, hopefully, avoid the drawbacks associated with the polar extremes of the interventional axis, from doing nothing to performing invasive surgery. The present paper was designed to outline the evolution of minimally invasive techniques for the application of botulinum chemodenervation that have been utilized in a referral practice that specializes in treating Thoracic Outlet Syndrome.

In recent years, botulinum toxin injections have been used for treating conditions characterized by muscle pain and dystonia (1-15). When botulinum toxin is injected into certain regions, targeting procedures using only surface landmarks may be sufficiently accurate and safe. However, for deeper targets with subjacent non-boney endpoints, where vulnerable soft tissue structures reside, and in regions where the inadvertent spread of toxin may pose increased risk, complementary targeting technologies have been used to improve accuracy and safety including electromyography, fluoroscopy and ultrasonography $(13,15-20)$. For botulinum chemodenervation in patients with Thoracic Outlet Syndrome and in other patients requiring the targeting of cervical muscles, specific risks include inadvertent chemodenervation of subjacent swallowing muscles with resultant dysphagia and excessive weakening of adjacent neck and shoulder muscles with undesirable consequences for posture and movement. More uncommon complications would include dysphonia, pneumothorax, vascular penetration with resultant hematoma, and brachial plexus injury. Published accounts of side effects such as dysphagia has varied from as low as 7 percent to over 30 percent $(1-11,16)$.

Although some of the side effects such as dysphagia may result from diffusion across tissue planes or from the systemic effects of toxin, inadvertent direct injection or bulk flow of toxin within tissue planes are additional mechanisms causing excessive toxin to reach undesired targets. Furthermore, precise targeting at the sites of toxin action closer to the innervation zones of muscle rather than in muscle tendon or distal muscle locations is likely to allow the use of very low doses, further reducing the chances of significant diffusion to subjacent tissues. The present study reports on a clinical experience using a technique that was designed to address the above considerations by combining electromyography with either ultrasonography or fluoroscopy, used within the controlled conditions of an outpatient surgical center. The clinical protocol was designed to minimize complications without sacrificing efficacy by using a precise targeting technique and minimal doses of botulinum toxin type $A$ (Botox).

In recent years, the availability of portable ultrasound devices with unobtrusive probes has facilitated the application of ultrasound technology for guidance in injection procedures in and around the brachial plexus $(21,22)$. Based on these previously published applications of ultrasonography, it was expected, in the present study, that an ultrasound based protocol would compare favorably to a fluoroscopically based approach that had been the subject of a previously reported experience. It was also expected that, after demonstrating the equivalency of outcomes with the two different imaging techniques, fluoroscopy could be replaced by ultrasonography in order to reduce costs and potential radiation exposure hazards.

\section{Methods}

All patients had cervical brachial pain and limb paresthesias worsened with overhead arm exercise. Subjects demonstrated temporary relief of symptoms with a selective block of the innervation to scalenes, pectorals, and subclavius muscles $(15,23)$. Cervical MRI scans, electromyography, and nerve conduction studies were used to exclude cases of cervical radiculopathy or other peripheral nerve compression syndromes. Patients were given the diagnosis of Thoracic Outlet Syndrome based on the above clinical findings. All of these patients had evidence of excessive muscle activity resulting in tight, tender muscles, cervical dystonia, and electromyographic evidence of excessive motor unit activity during attempted muscle relaxation in scalene, subclavius, or pectoralis minor muscles. All patients had failed a course of physical therapy including postural training, active stretching exercises and ergonomic adjustments. All subjects had a stable clinical syndrome for at least 2 years.

Botulinum toxin type A (Botox) was used for all subjects. Informed consent was obtained in all patients including the use of a document that included detailed information about possible botulinum side 
effects including dysphagia, dysphonia, pneumothorax, and inadvertent muscle weakness. The study was reviewed by the institutional review board and was in compliance with applicable IRB regulations as well as HIPAA requirements.

Patients were examined pre-operatively, at one month post-operatively, and 6 months post-operatively; patients responded to a numerical pain scale on each occasion. A good outcome was noted if there was a 50 percent or greater reduction in numerical pain scores as well as a decrease in the use of analgesics and a self-reported improvement in tasks requiring repetitive grasping, reaching and fingering. Self-reported improvements included specific examples which were individually meaningful such as the ability to blow dry or brush hair without help, the ability to type longer periods of time or the ability to carry groceries. A structured interview, using questions contained in a validated questionnaire, was administered to each patient post-operatively to detect possible dysphagia (24). Dysphonia or inadvertent muscle weakness was evaluated by interview and physical examination.

For the injection procedure, the patients were admitted to an outpatient surgical facility, an intravenous line was started, and patients were given light sedation with small doses of midazolam and fentanyl, if necessary, to maintain a fully awake, relaxed, and cooperative state. The patients were placed supine on a surgical table with the entire body tilted head up 30 degrees and the body was supported on a wedge to raise the targeted side. The head was stabilized with towel rolls and, if necessary to reduce movement in a particularly restless patient, 2-inch silk tape was used for head fixation. The targeted shoulder was depressed and stabilized by a surgical technician. Sterile technique was used throughout but the face was kept partially uncovered by the drapes to lessen any claustrophobic reactions.

In the earlier group of patients, fluoroscopic targeting was performed with routine $\mathrm{C}$-arm equipment and final placement was confirmed with injection of $1 / 2$ cc of iodinated contrast media (Isovue 200). After November 2003, fluoroscopy was replaced with intraoperative ultrasound using a 7.5 megahertz ultrasound probe attached to a portable ultrasound device. Before injection, a preliminary ultrasound examination was performed to look for evidence of subclavian artery aneurism, subclavian vein clot, positional compression of the subclavian artery, abnormal blebs of the lung, anomalous positions of the vertebral artery or other vessels, or anomalous positions and sizes of the scalene muscles and their location with respect to the brachial plexus.

After initial imaging and using sterile technique, a standard 25 gauge Teflon coated needle, modified by curving the tip, was advanced under imaging guidance to the muscle targets with simultaneous electromyographic monitoring. Prior to November 2001, the anterior scalene and middle scalene muscles were targeted with this technique. After November 2001, additional targets were added, including the subclavius and pectoralis minor muscles.

To minimize radiation exposure, fluoroscopic guidance was performed with repeated brief pulses so that total fluoroscopic times were kept below 15 seconds in all cases. For ultrasound guidance, there was continuous imaging until the needle tip was seen entering the mid belly of each muscle. The needle tip was kept in view at all times; small rotatory movements of the needle allowed for constant relocalization of the tip as it was moved into deeper structures. With both imaging modalities, constant electromyography monitoring was performed with visual and auditory output; this allowed for instantaneous confirmation of muscular penetration and for detection of inadvertent tip movement during release of the downward pressure from the ultrasound probe, patient movement, connection of syringes, and depression of syringe plungers. Syringes were not connected to the needles during the initial targeting to avoid potential levering action of the attached syringe and to allow a floating action of the needle so that it would not become displaced easily with deep breaths, coughs, or hiccups or other patient movements. To avoid increased dead space, there was no interposed tubing between the needle hub and syringe.

For the anterior scalene muscle 12 units of Botox were injected, for the middle scalene muscle 12 to 15 units were injected. Twenty-five units were injected into the subclavius and 35 units were injected into the pectoralis minor. All patients received an additional 75 to 100 units into painful and dystonic neck and shoulder muscles including the trapezius and levator scapula; the latter sites were targeted with surface landmarks and 27-gauge needles. For both ultrasonography targeted procedures and fluoroscopy guided procedures, total operative times were kept to below 20 minutes. 


\section{Results}

A total of 245 procedures were performed in 109 patients. Seventy-three of the procedures, prior to November of 2001, included targeting of the anterior and middle scalene muscles but not into the subclavius and pectoralis minor muscles. Subsequently, all procedures included the pectoralis minor and subclavius muscles. In 168 procedures, guidance utilized combined fluoroscopy and electromyography, whereas, after November of 2003, 77 procedures were targeted using combined ultrasonography and electromyography. All patients were analyzed in sequence without any drop outs. Although the different groups were derived from different time periods, the patients had been entered in sequence and there was no apparent difference in the treatment or evaluation of these patients according to the clinic routine.

After combined ultrasonography and electromyography there were no instances of dysphagia, undesired muscle weakness, dysphonia, pneumothorax, or any other side effects that might be attributed to missing targeted muscles or to inadvertent diffusion of toxin. After 2 procedures with combined fluoroscopy and electromyography, patients experienced dysphagia lasting several weeks. After 1 fluoroscopically guided procedure, a patient developed undesired weakness of an underlying shoulder muscle. The complication rate was $1.2 \%$ for dysphagia and $0.6 \%$ for undesired muscle weakness with an overall complication rate of $1.8 \%$. Comparing outcomes after fluoroscopic guidance with outcomes after ultrasonic guidance, there was no statistically significant difference in complication rates (Fisher exact $p=0.3206$ ). Although no systematic effort was made to document the development of paresthesias during needle placement with combined fluoroscopy and electromyography, the latter was not unusual in the experience of the authors. There were no instances of parethesias using ultrasonic guidance; the brachial plexus could be easily visualized in all cases so that the nerve elements could be avoided during needle penetrations.

For combined ultrasonography and electromyography, after 70 out of 77 procedures $(91 \%)$, there was a good outcome as defined in the methods section compared to 136 out of 168 after procedures ( $81 \%$ ) utilizing a combination of ultrasonography and electromyography (Fisher exact $p=0.331$ ). Procedures without targeting of the subclavius and pectoralis minor muscles had a good outcome in 50 out of 73 instances $(68 \%)$ which was significantly less than the 156 out of
$172(91 \%)$ with a good outcome when the subclavius and pectoralis minor sites were added (one tailed Fisher exact $p$ equals .0201). Overall, the duration of benefit was an average of $4.7+/-1.7$ months.

\section{Discussion}

Compared to previously published accounts (15), the present study demonstrates that, in a controlled environment, targeting techniques combining imaging guidance and electromyography can minimize complications while maintaining efficacy. The present study also demonstrated that ultrasonography is comparable to a technique utilizing fluoroscopy in reducing side effects due to inadvertent spread of botulinum toxin. These findings would suggest that ultrasonography may be considered as a replacement for fluoroscopy in performing selective muscle injections. Replacement of fluoroscopy may be considered desirable because of reduced radiation exposure to physician and patient. In addition, the relative cost of the 2 modalities favors ultrasound; ultrasonography based approaches avoid the additional personnel and equipment costs of fluoroscopy and the expense of contrast dye. Ultrasound in this study was entirely physician operated without any need for a fluoroscopy technician. Acquisition and leasing costs favor ultrasonography over fluoroscopy by a substantial margin; in this study, the ultrasound equipment costs approximately $\$ 40,000$ compared to more than 10 times that amount for the fluoroscopy machine. The ultrasound machine can also be operated in a small room, the fluoroscopy equipment has a much larger footprint.

It is apparent from this study that the complications of dysphagia and other undesired effects of inadvertent toxin spread are not inevitable consequences of chemodenervation procedures, even when toxin is injected in the scalene muscles, adjacent to muscles involved in swallowing and phonation. Precise targeting and the use of low doses are likely to be important factors in reducing undesired toxin effects. A controlled environment with protocols designed to reduce patient movement is likely to reduce the chances of needle tip displacement after targeting and during toxin injection. In the presently reported protocols, electromyography was used for confirming initial muscle penetration as well as for the detection of inadvertent needle tip displacement during the procedure. Although we did not systematically document the frequency of electromyographically detected inadvertent tip displacement, it was 
not uncommon for electromyography to detect such displacements. Whether or not electromyography contributes significantly to the low rates of dysphagia in this study, may not be the only justification for using this modality. Another potential factor justifying the use of electromyography includes the ability of this modality to show characteristic excessive motor unit activity that would confirm proper selection of clinical relevant dystonic muscles $(15,19)$. In certain patients, tight, tender muscles and a clinical condition of Thoracic Outlet Syndrome may be associated with excessive fibrosis of a muscle or with a cervical band (25); electromyography can distinguish excessive muscle contraction characteristic of dystonic muscles from passively contracted fibrotic muscles or fibrotic bands, avoiding unnecessary botulinum injection into such sites. Furthermore, in patients with repeat procedures, the presence of electromyographic patterns of reinnervation in targeted muscles confirms the accuracy and chemodenervation efficacy of prior botulinum injections.

Imaging data from the ultrasound examination performed at the time of the procedure is able to reveal additional findings that may be of clinical value, including whether targeted muscles are excessively atrophic, displaced or anomalous in location or depth, and how these muscles are juxtaposed with respect to normal or anomalous neural and vascular elements. In this study, rapid and accurate targeting was performed using ultrasound without inadvertent penetration of lungs, nerves or major blood vessels in any of the 77 procedures; this experience would support the concept that this modality can provide valuable targeting information in soft tissues in that cannot be easily obtained with other technologies. Although, after the presently reported fluoroscopically guided procedures, there were no instances of pneumothorax and no neurovascular injury was encountered, it is anticipated that such complications would be encountered on occasion because of the inability of the latter modality to visualize lung tissue or arterial structures, respectively. In contrast to the ultrasonography experience, fluoroscopy cannot image neural structures during needle insertion so that it was not unexpected for parethesias to occur during use of the latter modality.

Improvement in outcomes after adding the subclavius and pectoralis minor muscles to the target set could be explained on the basis of providing additional decompression at the costoclavicular space in patients with Thoracic Outlet Syndrome. Although scalene muscle relaxation may allow the first rib "floor" of the costoclavicular space to fall away from the overlying clavicle, it would be expected that further relaxation of muscles pulling down the clavicular "roof" of the costoclavicular space would provide additional decompression of the distal thoracic outlet. Toxin induced atrophy of the subclavius muscle may further decompress the costoclavicular space.

\section{Conclusion}

Compared to reports in the literature, the present study demonstrates that in a controlled setting, the use of combined imaging and electromyographic targeting techniques can minimize complications caused by inadvertent spread of toxin without sacrificing efficacy. Furthermore, the present study demonstrates that ultrasound may be considered a replacement for fluoroscopy when choosing an imaging modality for the guidance of deep muscle injections.

\section{References}

1 Brans JW, de B, I, Aramideh M, Ongerboer d, V, Speelman JD. Botulinum toxin in cervical dystonia: low dosage with electromyographic guidance. J Neurol 1995; 242:529-534.

2 Dubinsky RM, Gray CS, Vetere-Overfield $B$, Koller WC. Electromyographic guidance of botulinum toxin treatment in cervical dystonia. Clin Neuropharmacol 1991; 14:262-267.

3 Gelb DJ, Lowenstein DH, Aminoff MJ. Controlled trial of botulinum toxin injections in the treatment of spasmodic torticollis. Neurology 1989; 39 1:80-84. ankovic J, Schwartz K. Botulinum toxin injections for cervical dystonia. Neurology 1990; 40:277-280.

5 Kessler KR, Skutta M, Benecke R. Longterm treatment of cervical dystonia with botulinum toxin A: efficacy, safety, and antibody frequency. German Dystonia Study Group. J Neurol 1999; 246:26574.

7 Poewe W, Schelosky L, Kleedorfer B, Heinen F, Wagner M, Deuschl G. Treatment of spasmodic torticollis with local injections of botulinum toxin. One-year follow-up in 37 patients. J Neurol 1992;
239:21-25.

8 Racette BA, Gee-Minnich L, Perlmutter JS. Efficacy and safety of a new bulk toxin of botulinum toxin in cervical dystonia: a blinded evaluation. Clin Neuropharmacol 1999; 22:337-339.

9 Ranoux D, Gury C, Fondarai J, Mas JL, Zuber M. Respective potencies of Botox and Dysport: a double blind, randomised, crossover study in cervical dystonia. J Neurol Neurosurg Psychiatry 2002 April; 72 4:459-462.

10 Stell R, Thompson PD, Marsden CD. Botulinum toxin in spasmodic torticol- 
lis. J Neurol Neurosurg Psychiatry 1988 July; 51 7:920-3.

11 Anderson TJ, Rivest J, Stell R Steiger MJ, Cohen $\mathrm{H}$, Thompson PD, Marsden CD. Botulinum toxin treatment of spasmodic torticollis. J R Soc Med 1992 September; 85:524-529.

12 Trosch R. Botulinum toxin type A Botox in the treatment of refractory myofascial cervicothoracic pain: a prospective trial. Pain Med 2002 June; 3 2:173-174.

13 Lang AM. Botulinum toxin type B in piriformis syndrome. Am J Phys Med Rehabil 2004 ; 83 3:198-202.

14 Porta M, Maggioni G. Botulinum toxin BoNT and back pain. J Neurol 2004; 251 Suppl 1:115-l18.

15 Jordan SE, Ahn SS, Freischlag JA, Gelabert HA, Machleder HI. Selective botulinum chemodenervation of the scalene muscles for treatment of neurogenic thoracic outlet syndrome. Ann Vasc Surg 2000; 14:365-369.

16 Comella CL, Tanner CM, Foor-Hill L, Smith
C. Dysphagia after botulinum toxin injections for spasmodic torticollis: clinical and radiologic findings. Neurology 1992; 42:1307-1310.

17 Willenborg MJ, Shilt JS, Smith BP, Estrada RL, Castle JA, Koman LA. Technique for iliopsoas ultrasound-guided active electromyography-directed botulinum a toxin injection in cerebral palsy. J Pediatr Orthop 200; 22:165-168.

18 Trosch R. Fluoroscopic and electromyographic guided injection of the pirformis muscle with botulinum toxin type B. Pain Med 2002; 3:179.

19 Comella CL, Buchman AS, Tanner CM, Brown-Toms NC, Goetz CG. Botulinum toxin injection for spasmodic torticollis: increased magnitude of benefit with electromyographic assistance. Neurology 1992; 42:878-882.

20 Molloy FM, Shill HA, Kaelin-Lang A, Karp BI. Accuracy of muscle localization without EMG: implications for treatment of limb dystonia. Neurology
2002 March 12; 58 5:805-807.

21 Chan VW. Applying ultrasound imaging to interscalene brachial plexus block. Reg Anesth Pain Med 2003 July; 28:340-343.

22 De AJ, Sala-Blanch X. Ultrasound in the practice of brachial plexus anesthesia. Reg Anesth Pain Med 2002 January; 27 1:77-89.

23 Jordan SE, Machleder HI. Diagnosis of thoracic outlet syndrome using electrophysiologically guided anterior scalene blocks. Ann Vasc Surg 1998; 12:260264.

24 Wallace KL, Middleton S, Cook IJ. Development and validation of a self-report symptom inventory to assess the severity of oral-pharyngeal dysphagia. Gastroenterology 2000; 118:678-687.

25 Brantigan CO, Roos DB. Etiology of neurogenic thoracic outlet syndrome. Hand Clin 2004; 20:17-22 